\title{
Predicting perceived stress and resilience: the role of differentiation of self
}

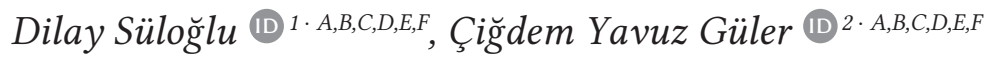 \\ 1: Üsküdar University, Istanbul, Turkey \\ 2: Fatih Sultan Mehmet Vakıf University, Istanbul, Turkey
}

\section{BACKGROUND}

Differentiation of self is a process that describes an individual's ability to act by separating emotions and thoughts at the internal level as well as the ability to be activated by maintaining the self in intense relationships through the establishment of a balance between autonomy and closeness at the level of interpersonal relations. The aim of this study was to determine the role of the differentiation of self - which is one of the basic concepts of the Bowen family systems theory - in predicting perceived stress and resilience.

\section{PARTICIPANTS AND PROCEDURE}

The research study group consisted of 423 individuals who were selected from a Turkish sample using the convenience sampling method. The study data were collected using a demographic form, the Differentiation of Self Inventory-Revised (DSI-R), the Perceived Stress Scale (PSS), and the Brief Resilience Scale (BRS).

\section{RESULTS}

The results indicate that as the level of differentiation increases, the perceived stress decreases, and resilience increases. Furthermore, differentiation of self is shown to predict $33 \%$ of the perceived stress level and $35.2 \%$ of the resilience level.

\section{CONCLUSIONS}

This study found that differentiation of self predicts the levels of stress that individuals experience and their resilience in stressful situations. The presented findings are meant to serve as a guide for mental health professionals working on coping with anxiety and stress, and increasing resilience in structuring the psychotherapy process.

\section{KEY WORDS}

Bowen family systems theory; differentiation of self; perceived stress; resilience 


\section{BACKGROUND}

\section{BOWEN FAMILY SYSTEMS THEORY AND DIFFERENTIATION OF SELF}

Bowen (1978) family systems theory considers the family, like all living systems, to be both a natural and an emotional unit. According to Bowen, the nature of problems is intergenerationally transmitted within the family system. An individual's problems are caused by the anxiety they experienced within the system and the problems that they have in their relationships. According to Kerr and Bowen (1988, pp. 54-55), the family is much more than an emotional unit - it is an emotional "sphere" because its members contribute and react at many different levels, involving the complexity of emotional stimuli. The emotional functioning of family members further affects the functions of each family member.

Differentiation of self is one of the basic concepts of Bowen (1978) family systems theory. Differentiation of self occurs at two levels: internal and interpersonal. It is a process that describes how an individual acts by separating their emotions and thoughts at the internal level. It also describes how it can be activated by establishing a balance between autonomy and intimacy through preserving one's self in intense relationships at the interpersonal level. Furthermore, differentiation of self refers to how the emotional reactivity level that a person develops in relation to their original family members, with an emphasis on the relationship with parents, is transmitted to new relationships (Titelman, 2008).

Skowron and Friedlander (1998) explain the internal and interpersonal indicators of differentiation of self using the following four factors: "I" position, emotional reactivity, fusion with others, and emotional cutoff. The "I" position can be described as the ability to protect an individual's self and reveal the said self when an individual is forced by others to behave differently and to present individual beliefs without being influenced by others (Bowen, 1978). Emotional reactivity includes the expression of existing anxiety toward oneself or someone else in the form of, for example, denial, attack, estrangement, and accusation (Titelman, 2008). Fusion with others can be expressed as the inability to differentiate one's self from another and being in an intertwined relationship. Emotional cutoff refers to an individual's emotional or physical distancing of oneself and to putting distance because they cannot cope with the anxiety within the system (Bowen, 1978; Kerr \& Bowen, 1988). Differentiation of self can be defined as an individual's ability to define one's self and the process of preserving the self's existence in intense relationships.

According to Bowen, separation from the symbiotic connection established within the original family is an important precursor of functionality. Differentiation seems to be a way of ensuring the emotional autonomy of an individual, revealing the self in a healthy manner and thus protecting oneself from the anxiety and stress caused by relationships. Another aspect of differentiation is characterized by having awareness of the behavioral patterns transmitted from past generations and not repeating these behaviors in one's own life. In contrast, being undifferentiated refers to a relationship-oriented functionality that constantly causes individuals to have chronic anxiety (Kerr \& Bowen, 1988).

\section{STRESS AND RESILIENCE}

One of the most highlighted concepts in Bowen's theory is the aforementioned chronic anxiety (Bowen, 1976; Kerr \& Bowen, 1988). Differentiation is regarded to be a form of human function, and Bowen states that anxiety increases as the level of differentiation decreases. Anxiety may appear as either the reason for or the result of existing problems. Furthermore, a distinction is made between acute and chronic anxiety. Acute anxiety occurs in a limited timeframe in response to real threats, while chronic anxiety usually occurs without a time limitation in response to imaginary threats. The higher the level of chronic anxiety in a relationship system, the greater the pressure on people's adaptability. The intensity of a person's anxious response to stress can disrupt their own functioning and/or that of people to whom they are emotionally attached. In such a case, neither this individual nor the system can be functional. An individual whose function is impaired as a result of chronic anxiety shows physical, emotional, or social symptoms. Symptom development is directly proportional to the amount of stress and anxiety experienced (Kerr \& Bowen, 1988).

Over the course of a lifetime, an individual frequently experiences relationship problems, career challenges, and events affecting the quality of life - life cycle changes, such as birth, divorce, and retirement, or traumatic life events, such as a natural disaster or the loss of a beloved person. Selye (1957) broadly defines stress as "non-specific response of the body to any demand." Stress is a phenomenon that disrupts an individual's biological or psychological balance (Selye, 1957). Situations leading to stress can constitute either a real danger or a situation that a person perceives as being potentially dangerous. While some physical effects, including blood pressure, headache, heart palpitations, and nausea, can be experienced in the case of stress, behavioral reactions, such as smoking, drinking alcohol, crying, or trembling, can also be observed in response to stress (Schneiderman et al., 2005). When presented with a stressful situation, the body responds by calling 
for the release of hormones that provide a burst of energy in order to help it cope with the current situation. These reactions are processes that occur automatically in an attempt to restore balance in both the body and environment (Lyon, 2000; Schneiderman et al., 2005; Selye, 1957). However, prolonged stress is known to have psychobiological consequences. Prolonged stress not only impairs the physical health of a person but also endangers their mental health (Fink, 2016; Lyon, 2000; Selye, 1957). The necessities of everyday life or traumatic events experienced are among the main causes of stress. The reactions of individuals in these moments of stress - i.e., how they cope with this stress - vary depending on their resilience (Schneiderman et al., 2005). Resilience is defined as the ability to recover and the power to return to normal life in cases in which an individual is exposed to a significant threat or negativity that interrupts either their development or life (Garmezy, 1993). Briefly, resilience is the ability of an individual to adapt to changes in their life, especially to stressful and traumatic situations (Garmezy, 1993; Gizir, 2007; Luthar et al., 2000).

Individuals react differently to the micro or macro traumas that they experience in their lifetimes and develop different coping strategies to deal with them. While some individuals can use their talents and capacities more effectively to more easily get out of stressful or depressive situations in which they find themselves as a result of their negative experiences, this process can be much longer for others, making it more difficult for them to return to their normal lives. This dynamic has paved the way for research studies that evaluate resilience and stress together (Bonanno et al., 2007; Ong et al., 2006).

\section{DIFFERENTIATION OF SELF, STRESS, AND RESILIENCE}

One of the prominent aspects of differentiation of self is the capacity of an individual to cope with stress in life (Kerr \& Bowen, 1988). Differentiated individuals are able to tolerate strong emotions even under challenging life conditions and to make decisions by considering their thoughts, thus coping with stress more effectively (Bowen, 1978; Rodríguez-González et al., 2019). In this regard, differentiation of self can be considered to be a significant factor affecting an individual's perspective and way of thinking (Skowron \& Friedlander, 1998). According to Bowen (1978), differentiation acts like a buffer against anxiety, stress, and psychological problems.

According to Kerr and Bowen (1988), less differentiated individuals experience anxiety more obviously because they can achieve only a very low level of emotional differentiation from their original family. In such cases, people around them keep asking,
"Why are you so anxious?" These less differentiated individuals are suffering from all the anxiety that surrounds them and are inclined to influence others with their own anxiety.

According to Skowron et al. (2004), the stress level and psychological adaptation of an individual depend on their ability to regulate emotional reactivity, to maintain relationships with others while avoiding emotional cutoffs, and also on their ability to have the "I" position in their relationships. A differentiated system includes an emotional reserve that is capable of adapting to stress. Intense and prolonged stress can exceed the limits of this reserve and psychological symptoms can occur. In relationships in which differentiation is low, the pressure of adaptation is so predominant and substantial that symptoms tend to be a constant feature of the relationship in order to maintain balance and harmony. In this case, as long as stress increases, symptoms also increase (Kerr \& Bowen, 1988).

In their empirical study, Murdock and Gore (2004) examine the correlation between differentiation and stress, revealing that more differentiated individuals can better cope with everyday life stress and are more successful in problem-solving. Cavaiola et al. (2012) examined the effects of differentiation of self on professional life, discovering that highly differentiated individuals report higher job satisfaction and lower interpersonal stress. Other researchers have reported that differentiation of self is positively correlated with differentiation in the workplace. In addition to these studies, many empirical studies that have tested the Bowen theory have found that differentiation of self is related to numerous psychological health variables (Biadsy-Ashkar \& Peleg, 2013; Jankowski \& Sandage, 2012; Rodríguez-González et al., 2019; Skowron et al., 2009; Yousefi et al., 2009) as well as to numerous psychological symptoms or problems (Bartle-Haring et al., 2002; Murdock \& Gore, 2004; Peleg-Popko, 2002; Peleg \& Rahal, 2012; Peleg \& Zoabi, 2014).

Although stress is a highly examined concept in mental health literature, few studies emphasize the significance of resilience for individuals under stress as a result of differentiation of self and other stressful conditions.

\section{PARTICIPANTS AND PROCEDURE}

\section{PARTICIPANTS}

This study was conducted with a total of 423 participants from a Turkish sample. Of these, 179 (35.4\%) were male, while $326(64.6 \%)$ were female. They were between 18 and 35 years of age, with an average of $27.83 \pm 5.16$. While $288(68.1 \%)$ of the participants were single, 135 (31.9\%) were married. With respect to education, 53 participants (12.5\%) were below high 
school level, 40 (9.5\%) had an associate degree, 261 $(61.7 \%)$ had an undergraduate degree, and 69 (16.4\%) had a graduate degree.

\section{DATA COLLECTION TOOLS}

Demographics form. A demographics form was prepared by the researchers for this study and it included information about the participants' gender, age, and marital status.

Differentiation of Self Inventory-Revised (DSI-R). The Differentiation of Self Inventory (DSI) measure was first developed by Skowron and Friedlander (1998). The DSI is a multidimensional differentiation scale that focuses on the intimate relationships of adults and on their existing relationships with their family of origin. The scale consists of 4 subscales with 43 items, which were later revised by Skowron and Schmitt (2003). The Turkish adaptation of the scale was made by Isik and Bulduk (2015). With this adaptation study, the DSI was revised to have a total of 20 items and consists of four subscales: emotional reactivity, emotional cutoff, fusion with others, and taking the "I" position. The high score obtained on the entire scale and its subscales indicates a high level of differentiation of self. The validity studies conducted supported the four-factor structure. The DSI-R internal consistency reliability coefficient was found to be $.81(\mathrm{DT}=.78, \mathrm{BP}=.75, \mathrm{BB}=.74, \mathrm{DK}=.77)$ for the entire scale, whereas the test-retest reliability coefficient that was obtained at an interval of 5 weeks was found to be $r=.74$. For this study, the reliability coefficient of the scale was calculated as .82 .

Perceived Stress Scale (PSS). The Perceived Stress Scale was developed by Cohen et al. (1983) and adapted into Turkish by Eskin et al. (2013). It consists of two subscales - Insufficient Self-Efficacy Perception and Perception of Stress/Discomfort - and aims to determine how stressful some situations are perceived to be in an individual's life. It consists of 14 items that use a 5-point Likert-type scale, ranging from 0 (never) to 4 (very often). Higher scores on the scale indicate higher levels of perceived stress. Eskin et al. (2013) examine the reliability and validity of three different forms of this scale with 14,10 , and 4 items in a Turkish sample. They find that all three forms of the scale are sufficiently reliable and that their internal consistency coefficients are $.84, .82$, and .66 for PSS-14, PSS-10, and PSS-4, respectively (Eskin et al., 2013). In addition, they point out that the perceived stress scores obtained from the 3 different PSS forms are positively correlated with life events and depression but negatively correlated with life satisfaction, self-esteem, and perceived social support scores (Eskin et al., 2013). A 14-item form was used in the present study, where the reliability coefficient of the scale was calculated as .87 .
Brief Resilience Scale (BRS). The Brief Resilience Scale was developed by Smith et al. (2008) in order to test the resilience of individuals. The BRS is a 5-point Likert-type, 6-item, one-scale, self-report style test tool. After the items that are on the scale and coded in reverse are translated, high scores indicate high resilience. The adaptation of the scale to a Turkish sample was performed by Dogan (2015). After exploratory and confirmatory factor analysis, it was found that the scale has a single-factor structure and the internal consistency coefficient of the scale was calculated as .83. Within the criterion-related validity context, it was found that there are positive correlations between the BRS and the Oxford Happiness Questionnaire, the Ego Resilience Scale, and Connor Davidson's Psychological Resilience Scale. In this study, the reliability coefficient of the scale was calculated as .87.

\section{PROCEDURE}

The data collection tools used in this research study were applied to volunteer participants aged 18 and above. After the study was approved by the University Ethics Committee, participants participated through an online questionnaire. Prior to participating, the researchers obtained consent from the participants.

\section{DATA ANALYSIS}

The SPSS-16 package program was used for statistical analysis during study result evaluation. The consistency of parameters with normal distribution was examined using the Kolmogorov-Smirnov test during data evaluation. Descriptive statistical methods (mean, standard deviation, number, and percentage) were used in data assessment, and variance analysis was used for comparisons of parameters showing normal distribution in quantitative data. Student's $t$-test was used for comparisons made between two groups. Pearson correlation analysis was performed in order to examine quantitative data relationships, while multiple regression analysis was performed to examine predictive power. Results were evaluated at a $95 \%$ confidence interval and significance was considered at the $p<.05$ level.

\section{RESULTS}

\section{EXAMINING THE DIFFERENTIATION OF SELF IN TERMS OF DEMOGRAPHIC VARIABLES}

Pearson correlation analysis was performed to examine the correlation between differentiation of self and age. According to the results, although no statistically significant difference was found between 
the total score of participant age and DSI-R $(r=.06$, $p>.05)$ and the levels of emotional reactivity $(r=-.01$, $p>.05)$, "I" position $(r=-.03, p>.05)$, and emotional cutoff $(r=.94, p>.05)$, a weak, statistically significant positive correlation was found between differentiation of self and fusion with others $(r=.11, p<.05)$. In other words, differentiation in commitment to others increases as age increases. An independent samples $t$-test was performed to evaluate whether differentiation of self could be differentiated by gender. Accordingly, no statistically significant difference was found in the subscales of "I" position $(t=-.52, p>.05)$ and emotional cutoff $(t=-.44, p>.05)$ with respect to the gender variable. A significant difference was found in favor of men in emotional reactivity subscale scores $(t=6.94, p<.01)$. In other words, differentiation at the emotional reactivity level is significantly higher for men than women. A significant difference was also found in favor of men in fusion with others subscale scores $(t=2.78, p<.01)$. In other words, differentiation at the fusion with others level is significantly higher for men than women. In addition, a significant difference was found in favor of men in the total DSI-R scores $(t=3.40, p<.01)$. In other words, the overall differentiation of self level is significantly higher for men than women. When the marital status variable was considered, no statistically significant difference was found in the total score of "I" position $(t=.62, p>.05)$, fusion with others $(t=-.35, p>.05)$, and differentiation of self in relation to the marital status variable $(p>.05)$. However, a significant difference was found in favor of married participants in emotional cutoff scale scores $(t=-2.56, p<.05)$. In other words, differentiation at the emotional cutoff level is significantly higher for married individuals than single ones. On the other hand, a significant difference was found in favor of single participants in emotional reactivity scale scores $(t=-2.04, p<.05)$. In other words, differentiation at the emotional reactivity level is significantly higher for single individuals than married ones.

\section{RESULTS RELATED TO THE PREDICTIVE POWER OF DIFFERENTIATION OF SELF FOR PERCEIVED STRESS}

Before analyzing the related predictive power of differentiation of self for perceived stress, the correlations between the two variables were revealed.

In Table 1, Pearson correlation analysis was used to examine the correlation between differentiation of self and perceived stress. According to the results of the analysis, a statistically significant, weak negative correlation was found between the emotional reactivity level of the participants and their stress and discomfort perception levels $(r=-.47, p<.001)$; between the "I" position and stress/discomfort perception levels $(r=-.29, p<.001)$; between the emotional cutoff and stress/discomfort perception levels $(r=-.32$, $p<.001)$; and between the fusion to others and stress/ discomfort perception levels $(r=-.31, p<.001)$. In other words, as the scores on the subscales of differentiation increase, the level of stress and discomfort perception decreases. However, when the correlation between perceived stress and insufficient self-efficacy was examined in accordance with the analysis results, a statistically significant, weak negative correlation was found between the emotional reactivity level of the participants and their stress and discomfort perception levels $(r=-.28, p<.001)$; between the "I" position and stress/discomfort perception levels $(r=-.46, p<.001)$; between emotional cutoff and stress/discomfort perception levels $(r=-.35$, $p<.001)$; and between fusion with others and stress/ discomfort perception levels $(r=-.34, p<.001)$. In other words, as the scores related to the subscales of differentiation increase, the level of insufficient selfefficacy perception decreases. When the total scores were considered, a statistically significant, moderate negative correlation was found between the scores obtained from the DSI-R and the perceived stress level $(r=-.58, p<.001)$.

\section{Table 1}

Evaluating the correlation between differentiation of self and perceived stress levels

\begin{tabular}{|c|c|c|c|c|c|c|}
\hline \multirow[t]{3}{*}{ Differentiation of Self Scale } & \multicolumn{4}{|c|}{ Perceived Stress Scale } & \multirow{2}{*}{\multicolumn{2}{|c|}{$\begin{array}{c}\text { Total score } \\
\text { of perceived stress }\end{array}$}} \\
\hline & \multicolumn{2}{|c|}{$\begin{array}{c}\text { Stress/discomfort } \\
\text { perception }\end{array}$} & \multicolumn{2}{|c|}{$\begin{array}{c}\text { Insufficient perceived } \\
\text { self-efficacy }\end{array}$} & & \\
\hline & $r$ & $p$ & $r$ & $p$ & $r$ & $p$ \\
\hline Emotional reactivity & -.47 & $<.001$ & -.28 & $<.001$ & & \\
\hline "l" position & -.29 & $<.001$ & -.46 & $<.001$ & & \\
\hline Emotional cutoff & -.32 & $<.001$ & -.35 & $<.001$ & & \\
\hline Fusion with others & -.31 & $<.001$ & -.34 & $<.001$ & & \\
\hline Total score & & & & & -.58 & $<.001$ \\
\hline
\end{tabular}


Table 2

Simple linear regression analysis for the effect of total Differentiation of Self Inventory-R scores on the total Perceived Stress Scale scores

\begin{tabular}{|c|c|c|c|c|c|c|}
\hline Predictive & Predicted & B & $\mathrm{SE}_{\mathrm{B}}$ & $\beta$ & $t$ & $p$ \\
\hline \multirow[t]{2}{*}{ Differentiation of self } & Constant & 108.08 & 2.17 & & 49.93 & $<.001$ \\
\hline & Perceived stress & -1.09 & 0.08 & -.58 & -14.40 & $<.001$ \\
\hline$R=.58$ & $R^{2}=.33$ & \multicolumn{3}{|c|}{$F(1,421)=207.44$} & \multicolumn{2}{|c|}{$p<.001$} \\
\hline
\end{tabular}

Table 3

Evaluating the correlation between the Differentiation of Self Inventory-R and the subscales of the Differentiation of Self Inventory- $R$ and the Brief Resilience Scale

\begin{tabular}{lcc}
\hline & \multicolumn{2}{c}{ Resilience Scale } \\
\cline { 2 - 3 } & $r$ & $p$ \\
\hline Differentiation of Self Scale & .59 & $<.001$ \\
Emotional reactivity & .49 & $<.001$ \\
"I" position & .45 & $<.001$ \\
Emotional cutoff & .28 & $<.001$ \\
Fusion with others & .44 & $<.001$ \\
\hline
\end{tabular}

Table 2 presents the results of simple linear regression analysis with respect to whether the total DSI-R scores affected the total PSS scores, in other words, how much the differentiation of self scale scores predicted the perceived stress scale scores. Accordingly, it was revealed that the DSI-R scores affected the total PSS scores $(F(1,421)=207.44, p<.001)$. The DSI-R scores were found to predict $33 \%$ of the total PSS scores. In other words, $33 \%$ of perceived stress is explained by differentiation of self.

\section{RESULTS RELATED TO THE PREDICTIVE POWER OF DIFFERENTIATION OF SELF FOR RESILIENCE}

Before analyzing the results related to the predictive power of differentiation of self for resilience, the correlations between the two variables were assessed.

Table 3 presents the results of the Pearson correlation analysis examining the relationship between the DSI-R and the subscales of the DSI-R and the BRS. When the analysis results were evaluated in terms of the differentiation of self subscales, a statistically significant, weak positive correlation was found between emotional reactivity and resilience levels of the participants $(r=.49, p<.001)$; between the "I" position and resilience levels $(r=.45, p<.001)$; between the emotional cutoff and resilience levels $(r=.23$, $p<.001)$; and between fusion to others and resilience levels $(r=.44, p<.001)$. In other words, as the DSI$\mathrm{R}$ subscale scores of individuals increase, their resilience also increases. On the other hand, a statistically significant, strong positive correlation was observed between the participants' total differentiation of self score and their resilience $(r=.59, p<.001)$. In other words, as the differentiation of self level of the participants increases, their resilience level also increases.

\section{RESULTS RELATED TO THE INFLUENCE OF THE DIFFERENTIATION OF SELF INVENTORY-R ON THE PERCEIVED STRESS SCALE AND BRIEF RESILIENCE SCALES}

Table 4 presents the results of the simple linear regression analysis regarding whether the total DSI-R scores affected the total BRS scores - i.e., to what extent the DSI-R scores predict the BRS scores. It was found that the DSI-R scores affected the BRS scores $(F(1,421)=228.51, p<.001)-$ they predicted $35.2 \%$ of the resilience scale scores. In other words, $35.2 \%$ of resilience is explained by differentiation of self.

\section{DISCUSSION}

The aim of this study was to determine the predictive power of differentiation of self for perceived stress and resilience. To do so, the study examined whether differentiation of self could be differentiated in accordance with age, gender, and marital status variables. It was observed that the differentiation of older participants in comparison to younger participants only increases in relation to the fusion with others subscale. Although Bowen (1978) had stated that younger individuals are just as capable of defining their selves and of acting independently as older ones, Skowron and Friedlander (1998) revealed - in line with the findings of the present study - that younger individuals experience less differentiation in terms of fusion with others and that no differentiation is observed in terms of the "I" position. Isik and Bulduk (2015), in their study examining the psycho- 
Dilay Süloğlu, Çiğdem Yavuz Güler

Table 4

Simple linear regression analysis for the influence of the Differentiation of Self Inventory-R on Brief Resilience Scale scores

\begin{tabular}{lcccccc}
\hline Predictive & Predicted & $\mathrm{B}$ & $\mathrm{SE}_{\mathrm{B}}$ & $\beta$ & $t$ & $p$ \\
\hline Differentiation of self & Constant & 50.33 & 1.90 & & 26.44 & $<.001$ \\
& Resilience & 1.48 & 0.10 & .59 & 15.12 & $<.001$ \\
$R=.59$ & $R^{2}=.35$ & & $F(1,421)=228.51$ & $p<.001$ \\
\hline
\end{tabular}

metric features for a Turkish sample, found that differentiation of self does not differ with respect to age. Similarly, Yavuz Güler and Karaca (2021) examined the rumination and emotion regulation power of differentiation of self and concluded that differentiation of self is not related to age.

When the results of the present study were evaluated in terms of the gender variable, it was observed that the differentiation at emotional reactivity and fusion with others levels is significantly higher for men than women. When evaluated in terms of the total differentiation of self score, the latter was also found to be significantly higher for men than women. Skowron et al. (2004) also examined the correlations between stress, differentiation of self, and psychological adaptation in university students and found that gender had no effect. Yavuz Güler and Karaca (2021) found that differentiation of self was similar for both male and female participants in terms of "I" position, emotional cutoff, and fusion to others, while the scores of men were significantly higher than the scores of women in terms of differentiation of emotional reactivity - one subscale of the DSI-R. In their study, Skowron and Friedlander (1998) developed a test tool for differentiation of self on which women obtained a higher level of differentiation in terms of emotional reactivity in comparison to men. In a Spanish case study, Rodríguez-González et al. (2015) found that the total differentiation level was significantly higher for men than women. Their results also revealed that men differentiated significantly higher in comparison to women in terms of fusion with others and emotional reactivity when evaluated in terms of subscales. Isik and Bulduk (2015) reported that the results of their study on Turkish sample indicated that women are more emotionally reactive, more dependent on others in their relationships, and have more difficulty in achieving the "I" position in relationships than men.

Emotional reactivity can be expressed as the behavioral and physiological manifestation of anxiety in response to another person, either in an acute or chronic form. Behaviors such as self-accusation, accusing others, attacking, denial, and resentment can be evaluated as manifestations of emotional reactiv- ity. These results suggest that women differentiate in a more difficult way than men, especially in terms of differentiation, fusion with others, and emotional reactivity. When evaluated by culture, the findings can be explained by the fact that the Turkish family system allows males to be separated from the root family more easily and that they act more cautiously towards girls.

When evaluated in terms of marital status, the results revealed that single individuals experience more emotional cutoff, while married ones experience more emotional reactivity. In a Turkish case study, Yavuz Güler and Karaca (2021) noted that differentiation of self does not differ depending on the romantic relationship variable; however, those who have children are more emotionally reactive. Accordingly, it can be noted that interaction from the original family to the nuclear family increases and differentiation becomes difficult in terms of emotional reactivity. Less differentiation experienced by single individuals in terms of emotional cut off suggests that, when they cannot solve their problems with the original family, they are able to choose the strategy of moving away more easily. The results of the studies that examined differentiation of self in terms of demographic variables vary in the literature. This situation reveals that more empirical studies, in general, and cross-cultural studies, in particular, are required in order to address demographic differences or similarities related to the differentiation concept of Bowen family systems theory.

The present study revealed that as the level of differentiation increases, the perceived stress level decreases and resilience increases. According to the results, differentiation of self predicts $33 \%$ of the perceived stress level while predicting $35.2 \%$ of the resilience level. According to Kerr and Bowen (1988), the level of differentiation of self is equivalent to an individual's adaptation capacity. When chronic anxiety increases, adaptation capacity decreases. Adaptation capacity can be expressed as the capacity of individuals to regulate their anxiety and emotional reactivity. The anxiety experienced when an individual is between individuality, togetherness, emotions, and thoughts increases the stress level of that individual 
in life (Bowen, 1978). When differentiation is visible - in other words, when an individuals is able to balance their emotions and thoughts, individuality and togetherness - then anxiety decreases and an individual can have more functional reactions to stressful events.

Murdock and Gore (2004) carried out a study with 119 university students and their results are consistent with those of the present research. Differentiation of self and perceived stress were found to explain $48 \%$ of psychological symptoms, while differentiation was also found to moderate the relationship between stress and psychological symptoms. Bartle-Haring et al. (2002) noted that individuals who have less differentiation in terms of emotional reactivity experience more stressful events and have more psychological symptoms. On the other hand, in a study examining the relationships between stress, differentiation of self, and psychological adaptation in university students, Skowron et al. (2004) found that as differentiation increased, stress decreased and psychological adaptation increased. In addition, differentiation of self was shown to play a mediator role between stress and psychological adaptation (Skowron et al., 2004). Choi and Murdock (2017) examined the relationship between interpersonal conflict and depression in a study that included 260 individuals and observed that an outward manifestation of an individual's anger has a positive effect on the relationship between emotional reactivity and interpersonal conflict even if the stated manifestation was partial. However, they also found that outward revelation of anger is associated with emotional cutoff and depression (Choi \& Murdock, 2017). Peleg (2014) also examined the relationship between stressful life events in childhood, differentiation of self during adulthood, and intergenerational triangulation. Peleg's (2014) research revealed that the level of stressful events that occurred during childhood and adolescence, in both genders, is positively correlated with the level of dependency on others and intergenerational triangulation, which are two subscales of differentiation of self. Furthermore, the level of positive life events experienced by an individual during childhood was shown to be negatively correlated with the level of emotional reactivity and emotional cutoff, another two subscales of DSI-R. In the research, family members who experienced highly stressful events were found to experience less differentiation of self and, therefore, they were at risk (Peleg, 2014). Similarly, in a study on a sample of Korean college students, Moon and Kim (2021) found that differentiation of self has a partial mediating effect on the relationship between stress and depression and that differentiation of self is an important factor in stress and depression.

Many other studies in the existing literature have shown that differentiation of self positively affects the mental health of individuals. A few of these studies emphasized the relationship between differentiation of self and resilience. For instance, Sadeghi et al. (2020) - in a study with 300 adolescents in Khoramabad, Lorestan - found that higher scores of differentiation are related to greater resilience and hope - as are the scores on "I" position, emotional cutoff, and fusion with others. In a study aiming to increase resilience by teaching differentiation of self, Shokri and Mehrinia (2020) analyzed 30 mothers whose children had ADHD. They taught a 15-people experimental group about differentiation of self in a 10-session program, while no intervention was made with a 15-people control group. The results showed that teaching differentiation of self significantly influences resilience and feelings of entrapment in mothers of children with ADHD.

The results of the current study indicated that differentiation of self predicts the stress experienced by individuals and their resilience in stressful situations. The results of this study are expected to guide mental healthcare professionals who work on anxiety, stress, coping with stress, and increasing resilience in relation to structuring the psychotherapy process.

According to Kerr and Bowen (1988), differentiation is a way of thinking that expresses the way of being rather than a therapeutic technique. Providing a higher level of differentiation means increasing the emotional differentiation capacity of a person. Differentiation is associated with changes in the way an individual thinks. Such changes reveal themselves in the ability of an individual to make emotional contact with difficulties without preaching about what others should do, without rushing to fix the problem, or without pretending to be emotionally isolated and unaffected. This situation means neither moving away from the emotion nor acting with thought. Differentiation refers to the ability to act with an awareness of both emotions and thoughts. Differentiation is a matter of balance. When balance is found, individuals become more resistant to stressful living conditions and can take better care of their individuality while maintaining the harmony of togetherness despite pressures from others. By studying differentiation during the psychotherapy process, an individual can be made more functional in terms of coping with stress. In this regard, differentiation should be seen not only as a way of treating symptoms but also as a preventive mental health journey.

\section{CONCLUSIONS, LIMITATIONS, AND FUTURE DIRECTIONS}

The findings of this study have shown that differentiation significantly affects the perceived stress and resilience in challenging life events. Although the relationship between differentiation and stress 
has clearly been demonstrated in different cultures and different populations in the literature, the importance of this study relates to the fact that it has demonstrated that resilience, which is an important dynamic for coping with stress, is predicted by differentiation of self.

The findings of this study were obtained using regression analysis. Although this can be considered to be a limitation because there is no existing study in the literature that shows the relationship between differentiation of self and resilience, this study is expected to become a reference point for future mediation modeling studies. In subsequent studies, variables that mediate the relationship between differentiation of self and resilience can be determined. On the other hand, although the Bowen family systems theory on stress has been confirmed by studies in many different cultures, it is clear that the findings regarding the predictor of resilience also need to be tested in other individualistic and collectivistic cultures. The fact that the sample of this study consisted of individuals 18 to 35 years of age can be considered as a limitation of the study. Hence, future studies could repeat the research protocol outlined here on a sample of individuals who are above the age of 35 . The sample in this research study comprised a normal population. Therefore, the results can additionally be tested in a clinical sample in the future.

Although it has empirically been determined in the literature that differentiation of self is associated with many mental health dynamics, both positive and negative, little effort has been made to address this issue in clinical studies. This study showed that working on the differentiation of an individual during the psychotherapy process could contribute to a decrease of that individual's perceived stress and an increase in their resilience. We believe that the results presented here can benefit mental health professionals and encourage them to work on differentiation of self during the psychotherapy process with their clients when they work on coping with stress and increasing resilience.

\section{RefERENCES}

Bartle-Haring, S., Rosen, K. H., \& Stith, S. M. (2002). Emotional reactivity and psychological distress. Journal of Adolescent Research, 17, 568-585. https://doi.org/10.1177/074355802237464

Biadsy-Ashkar, A., \& Peleg, O. (2013). The relationship between differentiation of self and satisfaction with life amongst Israeli women: a cross cultural perspective. Health, 5, 1467-1477. https://doi. org/10.4236/health.2013.59200

Bonanno, G. A., Galea, S., Bucciarelli, A., \& Vlahov, D. (2007). What predicts psychological resilience after disaster? The role of demographics, resources, and life stress. Journal of Consulting and Clinical Psychology, 75, 671-682. https://doi.org/10.1037/0022006X.75.5.671

Bowen, M. (1976). Theory in the practice of psychotherapy. In P. J. Guerin, Jr. (Ed.), Family therapy: Theory and practice (pp. 42-90). Garner Press.

Bowen, M. (1978). Family therapy in clinical practice. Aronson.

Cavaiola, A. A., Peters, C., Hamdan, N., \& Lavender, N. J. (2012). Differentiation of self and its relation to work stress and work satisfaction. Journal of Psychological Issues in Organizational Culture, 3, 7-20. https://doi.org/10.1002/jpoc.20092

Choi, S. W., \& Murdock, N. L. (2017). Differentiation of self, interpersonal conflict, and depression: The mediating role of anger expression. Contemporary Family Therapy, 39, 21-30. https://doi.org/10.1007/ s10591-016-9397-3

Cohen, S., Kamarck, T., \& Mermelstein, R. (1983). A global measure of perceived stress. Journal of Health and Social Behavior, 24, 385-396. https://doi. org/10.2307/2136404

Doğan, T. (2015). Kısa psikolojik sağlamlık ölçeği'nin Türkçe uyarlaması: Geçerlik ve güvenirlik çalışması [Adaptation of the Brief Resilience Scale into Turkish: a validity and reliability study]. The Journal of Happiness \& Well-Being, 3, 93-102.

Eskin, M., Harlak, H., Demirkıran, F., \& Dereboy, Ç. (2013). Algılanan stres ölçeğinin Türkçeye uyarlanması: güvenirlik ve geçerlik analizi [Adaptation of the Perceived Stress Scale into Turkish: a reliability and validity analysis. New/Yeni Symposium Journal, 51, 132-140.

Fink, G. (2016). Stress, definitions, mechanisms, and effects outlined: Lessons from anxiety. In G. Fink (Ed.), Stress: Concepts, cognition, emotion, and behavior (pp. 3-11). Academic PrGarmezy, N. (1993). Children in poverty: Resilience despite risk. Psychiatry, 56, 127-136. https://doi.org/10.1080/00332 747.1993.11024627

Gizir, C. (2007). Psikolojik sağlamlık, risk faktörleri ve koruyucu faktörler üzerine bir derleme çalışması [A review study on psychological resilience, risk factors and protective factors]. Türk Psikolojik Danışma ve Rehberlik Dergisi, 3, 113-128.

Işık, E., \& Bulduk, S. (2015). Psychometric properties of the Differentiation of Self Inventory-Revised in Turkish adults. Journal of Marital and Family Therapy, 41, 102-112. https://doi.org/10.1111/jmft.12022

Jankowski, P. J., \& Sandage, S. J. (2012). Spiritual dwelling and well-being: The mediating role of differentiation of self in a sample of distressed adults. Mental Health, Religion \& Culture, 15, 417434. https://doi.org/10.1080/13674676.2011.579592

Kerr, M. E., \& Bowen, M. (1988). Family evaluation. WW Norton \& Company.

Luthar, S. S., Cicchetti, D., \& Becker, B. (2000). The construct of resilience: a critical evaluation and 
guidelines for future work. Child Development, 71, 543-562. https://doi.org/10.1111/1467-8624.00164

Lyon, B. L. (2000). Stress, coping, and health. In V. H. Rice (Ed.), Handbook of stress, coping, and health: Implications for nursing research, theory, and practice (pp. 3-23). Sage Publications.

Moon, W. H., \& Kim, J. Y. (2021). Stress and depression in the Korean college students: Mediated effects of self-differentiation and self-efficacy. In A. Bhoi, P. Mallick, C. M. Liu, \& V. Balas (Eds.), Bio-inspired neurocomputing. Studies in computational intelligence (pp. 151-161). Springer.

Murdock, N. L., \& Gore, P. A. (2004). Stress, coping, and differentiation of self: a test of Bowen theory. Contemporary Family Therapy, 26, 319-335. https://doi.org/10.1023/b:coft.0000037918.53929.18

Ong, A. D., Bergeman, C. S., Bisconti, T. L., \& Wallace, K. A. (2006). Psychological resilience, positive emotions, and successful adaptation to stress in later life. Journal of Personality and Social Psychology, 91, 730-749. https://doi.org/10.1037/00223514.91.4.730

Peleg, O. (2014). The relationships between stressful life events during childhood and differentiation of self and intergenerational triangulation in adulthood. International Journal of Psychology, 49, 462-470. https://doi.org/10.1002/ijop.12054

Peleg, O., \& Rahal, A. (2012). Physiological symptoms and differentiation of self: a cross-cultural examination. International Journal of Intercultural Relations, 36, 719-727. https://doi.org/10.1016/j. ijintrel.2012.04.001

Peleg, O., \& Zoabi, M. (2014). Social anxiety and differentiation of self: a comparison of Jewish and Arab college students. Personality and Individual Differences, 68, 221-228. https://doi.org/10.1016/j. paid.2014.04.032

Peleg-Popko, O. (2002). Bowen theory: a study of differentiation of self, anxiety and physical symptoms. Contemporary Family Therapy, 24, 355-369. https://doi.org/10.1023/a:1015355509866

Rodríguez-González, M., Schweer-Collins, M., Skowron, E. A., Jódar, R., Cagigal, V., \& Major, S. O. (2019). Stressful life events and physical and psychological health: Mediating effects of differentiation of self in a Spanish sample. Journal of Marital and Family Therapy, 45, 578-591. https://doi.org/ 10.1111/jmft. 12358

Rodríguez-González, M., Skowron, E. A., Cagigal de Gregorio, V., \& Muñoz San Roque, I. (2015). Differentiation of self, mate selection, and marital adjustment: Validity of postulates of Bowen theory in a Spanish sample. The American Journal of Family Therapy, 44, 11-23. https://doi.org/10.1080/0192 6187.2015.1099415

Sadeghi, M., Barahmand, U., \& Roshannia, S. (2020). Differentiation of self and hope mediated by resilience: Gender differences. Canadian Journal of Fam- ily and Youth, 12, 20-43. https://doi.org/10.29173/ cjfy 29489

Schneiderman, N., Ironson, G., \& Siegel, S. D. (2005). Stress and health: Psychological, behavioral, and biological determinants. Annual Review of Clinical Psychology, 1, 607-628. https://doi.org/10.1146/annurev.clinpsy.1.102803.144141

Selye, H. (1957). Lathyrism. Revue Canadienne de Biologie, 16, 1-82.

Shokri, L., \& Mehrinia, F. (2020). Effectiveness of self-differentiation training on psychological resilience and feeling entrapment in the mothers of the children with attention deficit and hyperactivity disorder (ADHD). Journal of Psychological Science, 19, 1031-1040.

Skowron, E. A., \& Friedlander, M. L. (1998). The Differentiation of Self Inventory: Development and initial validation. Journal of Counseling Psychology, 45, 235-246. https://doi.org/10.1037/0022-0167.45.3.235

Skowron, E. A., \& Schmitt, T. A. (2003). Assessing interpersonal fusion: Reliability and validity of a new DSI fusion with others subscale. Journal of Marital and Family Therapy, 29, 209-222. https:// doi.org/10.1111/j.1752-0606.2003.tb01201.x

Skowron, E. A., Stanley, K. L., \& Shapiro, M. D. (2009). A longitudinal perspective on differentiation of self, interpersonal and psychological well-being in young adulthood. Contemporary Family Therapy, 31, 3-18. https://doi.org/10.1007/s10591-008-9075-1

Skowron, E. A., Wester, S. R., \& Azen, R. (2004). Differentiation of self mediates college stress and adjustment. Journal of Counseling \& Development, 82, 69-78. https://doi.org/10.1002/j.1556-6678.2004. tb00287.x

Smith, B. W., Dalen, J., Wiggins, K., Tooley, E., Christopher, P., \& Bernard, J. (2008). The Brief Resilience Scale: Assessing the ability to bounce back. International Journal of Behavioral Medicine, 15, 194-200. https://doi.org/10.1080/10705500802222972

Titelman, P. (2008). The concept of the triangle in Bowen theory: an overview. In P. Titelman (Ed.), Triangles: Bowen family systems theory perspectives (pp. 3-61). Routledge.

Yavuz Güler, Ç., \& Karaca, T. (2021). The role of differentiation of self in predicting rumination and emotion regulation difficulties. Contemporary Family Therapy, 43, 113-123. https://doi.org/10.1007/ s10591-020-09559-1

Yousefi, N., Etemadi, O., Bahrami, F., Fatehezade, M. A., Ahmadi, S. A., \& Beshlideh, K. (2009). Structural relationships between self-differentiation and subjective wellbeing, mental health and marital quality "fitting" Bowen's theory. Iranian Journal of Psychiatry and Behavioral Sciences, 3, 4-14. 